\title{
Combined autologous cellular cardiomyoplasty with skeletal myoblasts and bone marrow cells in canine hearts for ischemic cardiomyopathy
}

\author{
Imran A. Memon, MBBS, Yoshiki Sawa, MD, Shigeru Miyagawa, MD, Satoshi Taketani, MD, and Hikaru Matsuda, MD
}

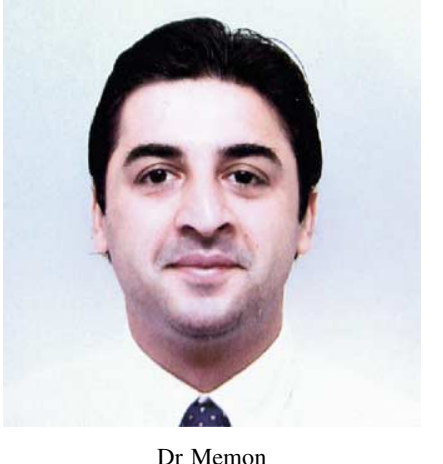

From the Osaka University Graduate School of Medicine, Department of Surgery E1, Division of Cardiovascular Surgery, Osaka, Japan.

Supported by the 1st Department of Surgery, Osaka University.

Received for publication Aug 17, 2004; revisions received Jan 4, 2005; accepted for publication Feb 4, 2005.

Address for reprints: Hikaru Matsuda, MD, Osaka University Graduate School of Medicine, Department of Surgery E1, Division of Cardiovascular Surgery, 2-2 Yamadaoka, Suita, Osaka, Japan (E-mail: memon@ surg1.med.osaka-u.ac.jp)

J Thorac Cardiovasc Surg 2005;130:646-53 $0022-5223 / \$ 30.00$

Copyright $\odot 2005$ by The American Association for Thoracic Surgery

doi:10.1016/j.jtcvs.2005.02.024
Objectives: Cellular cardiomyoplasty with isolated skeletal myoblasts and bone marrow mononuclear cells is an encouraging therapeutic strategy for heart failure. We investigated the achievements accomplished with combined cell therapy of skeletal myoblast and bone marrow mononuclear cell transplantation to the ischemic canine myocardium.

Methods: Autologous skeletal myoblasts $\left(1 \times 10^{8}\right)$ and autologous bone marrow mononuclear cells $\left(3 \times 10^{6}\right)$ were injected directly into the damaged myocardium of canine hearts that had undergone 2 weeks of left anterior descending coronary artery ligation. Treatment groups were as follows: skeletal myoblasts plus bone marrow mononuclear cells (combined cell therapy, $n=4)$, myoblasts $(n=4)$, bone marrow mononuclear cells $(n=4)$, and medium only $(n=4)$. In similarly designed supporting experiments, angiogenic factor expression was evaluated by enzymelinked immunosorbent assay after cell transplantation in rat hearts that had undergone left anterior descending coronary artery ligation.

Results: Four weeks after cell implantation, echocardiography demonstrated better cardiac performance with reduced left ventricular dilation and significantly improved ejection fraction in the combined cell therapy group compared with that seen in the other groups (pretreatment, $37.7 \% \pm 1.1 \%$, vs combined cell therapy, $55.4 \%$ $\pm 8.6 \%$; myoblasts, $47.4 \% \pm 7.4 \%$; bone marrow mononuclear cells, $44.4 \% \pm$ $6.7 \%$; medium only [control], $34.4 \% \pm 5.4 \% ; P<.05$ ). A significantly high number of neovessels were observed in the group receiving combined cell therapy only (combined cell therapy, $45.5 \pm 12 \times 10^{2} / \mathrm{mm}^{2}$; myoblasts, $26.5 \pm 8 \times 10^{2} / \mathrm{mm}^{2}$; bone marrow mononuclear cells, $30.7 \pm 15 \times 10^{2} / \mathrm{mm}^{2}$; medium only [control], 7.1 $\left.\pm 1 \times 10^{2} / \mathrm{mm}^{2} ; P<.05\right)$. Immunostained sections expressed the skeletal specific marker myosin heavy chain, although they did not express the cardiac specific marker troponin T. Results of enzyme-linked immunosorbent assay showed the highest expression of vascular endothelial growth factor (combined cell therapy, 2.9 $\pm 0.7 \mathrm{ng} / \mathrm{g}$ tissue; myoblasts, $0.24 \pm 0.7 \mathrm{ng} / \mathrm{g}$ tissue; bone marrow mononuclear cells, $1.9 \pm 0.2 \mathrm{ng} / \mathrm{g}$ tissue; medium only [control], $0.19 \pm 0.004 \mathrm{ng} / \mathrm{g}$ tissue; $P<$ $.05)$ and hepatocyte growth factor in the combined cell therapy hearts.

Conclusions: Combined autologous cellular therapy induced both myogenesis and angiogenesis with enhancement of cardiac performance and reduction of cardiac remodeling, suggesting a capable strategy for treating severe ischemic cardiomyopathy clinically.

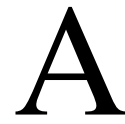
fter myocardial infarction, left ventricular (LV) chamber remodeling, scar formation, scar expansion, and continuous engulfment of the neighboring cardiomyocytes to the scar are ineluctable. LV heart failure and death is the unfortunate result because the heart lacks enough regenerative capacity. In this regard cellular cardiomyoplasty has been reported to have the potential of funda- 
mental regenerative capability and has already been introduced in clinical trials with skeletal myoblasts $(\mathrm{SMs})^{1}$ or bone marrow mononuclear cells (BM-MNCs), ${ }^{2}$ and results suggest that it is a relatively feasible and safe therapy. Autologous availability of these cells leaves all the ethical and immunologic issues behind.

The SMs are the mononucleated precursors to skeletal myofibers with graft generation capacity and have been widely selected for cell transplantation. Several reported works showed the limitation of scar area and attenuation of LV chamber with myoblast transplantation, leading to improved cardiac performance. ${ }^{3-5}$ Myoblast qualities, such as being myoregenerative and resistant to ischemia, ${ }^{6}$ with growth to millions in a short period of time and transformation to slow twitch manner, ${ }^{7}$ suits them for the clinical cellular cardiomyoplasty application.

In the ischemic myocardium myogenesis has been proposed for repairing the impaired heart functions and was achieved with SM cell transplantation; however, dominated regional blood flow defect in ischemic hearts, possible increased death of transplanted myoblasts, and restricted myoblast survival and growth are a few important limitations ${ }^{8}$ and must be addressed. Supply of angiogenic and growth factors enhances the efficacy of cellular cardiomyoplasty and appears to be beneficial to the transplanted cells. ${ }^{9}$ Given this body of evidence, we established the combined cell therapy (CCT) concept, myoangiogenesis, for heart regeneration and improvement in cardiac performance. $^{10}$

Bone marrow (BM) is a soft spongy tissue found within long bones. In addition to the blood cells, BM has multipotential mononuclear cells (MNCs) that contain various kinds of cell lineages, such as hematopoietic cells, fibroblasts, osteoblasts, and myogenic cells, as well as endothelial lineage. A subset of BM-MNCs demonstrates the differentiation to endothelial cells, ${ }^{11,12}$ whereas recent findings have confirmed the nontransdifferentiation of bone marrow cells (BMCs) into cardiomyocytes. ${ }^{13}$ Revascularization by BM-MNCs has the potential to improve ischemic area perfusion and cardiac function. ${ }^{14,15}$ BM-MNCs cause angiogenesis by releasing angiogenic factors, such as vascular endothelial growth factor (VEGF) and basic fibroblast growth factor (bFGF), ${ }^{11,15,16}$ and appeals as a suitable candidate for CCT.

In the present study we hypothesized that the myoblast transplantation might reremodel the heart, and the addition of BM-MNCs for angiogenesis and growth factor supply might enhance the effect of myoblast transplantation, leading to further improvement in cardiac performance as the autologous CCT.

\section{Methods}

Sixteen female canines (beagles, 7-8 months old; Oriental Yeast, Co Ltd, Osaka, Japan) weighing 8 to $10 \mathrm{~kg}$ were used in these experiments. Beagles were anesthetized with intravenous administration of sodium pentobarbital $(10 \mathrm{mg} / \mathrm{kg})$. Anesthesia was maintained with $1 \%$ sevoflurane inhalation after mechanical ventilation endotracheal intubation was performed. Left thoracotomy was performed through the fourth intercostal space, and a pericardiotomy was made. The left anterior descending coronary artery (LAD) was carefully dissected and ligated just distal of the first diagonal branch arising. Experimentally, myocardial infarction resulted in a less than $5 \%$ acute mortality rate within 24 hours of the operation, whereas the cell implantation procedure caused no additional animal deaths. The cultured autologous myoblasts and freshly isolated autologous BM-MNCs were transplanted. The pericardium and chest were closed in layers. In supporting experiments rat LADs (Wistar Lewis; 180-220 g; Seas Yoshitomi Ltd) were similarly ligated, and cells from syngeneic rats were transplanted. Rat model animals were merely used to evaluate the expression of growth factors. All animals received human care in compliance with the "Principles of laboratory Animal Care" formulated by the National Society for Medical Research and the "Guide for the Care and Use of Laboratory Animals" prepared by the institute of laboratory Animal Resource and published by the National institutes of Health (National Institutes of Health publication no. 86-23, revised 1985).

\section{Myoblast Isolation Procedure}

In canine experiments the autologous tibialis anterior muscle was removed (weight, 4-6 g) and placed in ice-cold normal saline solution. Connective tissues and tendons were carefully removed to minimize the presence of fibroblasts. The isolated muscle was minced and then treated with 1\% collagenase in Dulbecco's modified Eagle's medium (DMEM; Gibco BRL). Percoll (Percoll Amersham Biosciences) density centrifugation was carried out to obtain high-purity myoblast cultures. The cells were cultured in DMEM containing $20 \%$ fetal bovine serum and $1 \%$ antibiotics. First-week cultures contained $70 \%$ to $80 \%$ myoblasts, and at the time of cell transplantation, the cultures contained more than $60 \%$ myoblasts, as checked by means of desmin staining. Cultured myoblasts $\left(1 \times 10^{8}\right)$ were transplanted to canine hearts after 14 days of LAD ligation.

In rat experiments the myoblasts were isolated from the tibialis anterior muscle of syngeneic rats by using a similar protocol. Cultured myoblasts $\left(1 \times 10^{7}\right)$ were transplanted after 14 days of LAD ligation in rat hearts.

\section{Extraction of BM-MNCs}

The canines were anesthetized, and the BM $(\leq 20 \mathrm{~mL})$ was aspirated from the ileum with a BM harvest needle. About $20 \mathrm{~mL}$ of collected heparinized BM is mixed with saline solution suspended over the Percoll gradient (Lymphoprep Tube, Nycomed) in a sterile 50-mL plastic tube and centrifuged at $800 \mathrm{~g}$ for 20 minutes. The cells were collected from the interface, washed several times, and then suspended at a concentration of $3 \times 10^{6}$ in $1 \mathrm{~mL}$ of DMEM for transplantation. The cells were stored in the ice box for transplantation.

Rat BM was aspirated by isolating the femur and humerus bones, dissecting them, and flushing the BM with $5 \mathrm{~mL}$ of medium with a 21-gauge needle. BM-MNCs were collected by the protocol 
mentioned before. BM-MNCs $\left(3 \times 10^{5}\right)$ were transplanted to a single animal heart.

\section{BM-MNC/SM Transplantation Target Region}

Cultured autologous myoblasts and freshly extracted autologous BM-MNCs were separately suspended in 1 to $2 \mathrm{~mL}$ of DMEM solution and injected into the intramyocardium with a 26-gauge needle. For rat hearts, SMs and BM-MNCs were also separately suspended in 0.1 to $0.2 \mathrm{~mL}$ of DMEM and injected into the intramyocardium with a 30-gauge needle. Eight to 10 sites for each cell type, in close proximity to each other, were chosen for cell injection within and around the scarred area. The injection points were sutured to mark the locations. Maximum technical care was taken to avoid loss of cells. In CCT group hearts the ratio of transplanted myoblasts to BM-MNCs was around 33:1.

\section{Assessment of Cardiac Performance}

Dogs were anesthetized, and heart function was monitored at baseline ( 2 weeks after LAD ligation) and at 4 weeks after cell transplantation. Echocardiography was performed with an ultrasound machine (SONOS 5500, Agilent Technologies) equipped with an annular-array transducer operating at $12 \mathrm{MHz}$. Parasternal short-axis and long-axis views in $\mathrm{B}$ and $\mathrm{M}$ imaging modes were performed. The heart was imaged in the 2-dimensional mode in the short-axis views at the level of largest ventricular diameter, and then the systolic and diastolic diameter was determined. The LV end-systolic dimension was measured at the time of the most anterior systolic motion of the LV posterior wall, whereas diastolic measurements were made at the time of apparent maximal LV diastolic dimension. Global parameters, such as the LV enddiastolic diameters, end-systolic diameters, and ejection fraction $(\mathrm{EF})$, were determined. End-diastolic area (EDA) and end-systolic area (ESA) were also determined to evaluate the percentage of fractional area shortening (FAS) by using the following formula: $F A S(\%)=E D A-E S A \times 100(\%)$.

\section{Histopathology}

After 4 weeks of treatment, the canine hearts were removed and cryoprotected in liquid nitrogen and paraffin. Thin sections $(5 \mu \mathrm{m}$ in thickness) of each 5-mm short-axis slice were stained with hematoxylin and eosin for visualization of muscle and cellular structure and with Masson trichrome stain to delineate fibrous tissue. All infarcts were examined histologically to verify that the control dogs had transmural and myocyte-free defects. Rat hearts were not histologically examined. By random selection of 5 lowpower fields (40 $\times$ magnification) on light microscopy, the area of grafted myoblasts in the CCT and myoblast groups' infarcted scars was quantified by using the following formula: Length/2 $\times$ Width/2.

\section{Immunostaining for Neograft and Neovessels}

Paraffin blocks were sectioned, deparaffinized, and immunostained with the following antibodies: monoclonal antiskeletal myosin antibody to fast-twitch skeletal myosin heavy chain (MHC), (MY32, Sigma); monoclonal antimyosin (skeletal, slow) directed against skeletal slow MHC isoforms (NOQ7.5.4D, Sigma); and troponin $\mathrm{T}$ (Cardiac Isoform specific, Ab-1, mouse monoclonal antibody; NeoMarkers).
The nitrogen liquid frozen sections were immunostained with an antibody to von Willebrand factor-horseradish peroxidase (DAKO). Capillaries were counted by detecting the vascular endothelial cells. Ten different fields at $100 \times$ magnification were randomly selected, and the number of capillaries in each field was counted and expressed for results as the number of vessels per square millimeter.

\section{Angiogenic Factor Presence}

Syngeneic Lewis strain rats $(n=16)$ were used for enzyme-linked immunosorbent assay (ELISA) assessment of growth factors in heart tissue and cultured cell supernatant. The 2-week ligated LAD rats were used as CCT, myoblast, BM-MNC, and control groups, and the VEGF ELISA kit (R\&D Systems, Inc), bFGF ELISA kit (Chemikon Int Inc) and human growth factor (HGF) ELISA kit (Institute of Immunology, Tokyo) were used in our experiments. The same ratio numbers of SMs, BM-MNCs and SMs plus BMMNCs (coculture) were cultured individually for in vitro measurement of growth factors.

\section{Statistical Analysis}

All data are presented as means \pm SD. Differences in cardiac performance data were analyzed between the groups by the 2-way analysis of variance test. The other numerical data were analyzed by 1 -way analysis of variance. If a significant $F$ ratio was obtained, further analysis was carried out with the Turkey-Kramer post hoc test.

\section{Results}

\section{Global Cardiac Performance}

All 4 groups showed no significant difference in baseline (2 weeks after LAD ligation) echocardiographic measurements. The results after cell transplantation, however, showed diversity in all groups, as shown in Figure 1. EF measurements were $37.7 \%$ at baseline (average in 4 groups) and after 4 weeks measured as follows: CCT group, $55.3 \%$ $\pm 8.6 \%$; myoblast group, $47.4 \% \pm 7.4 \%$; BM-MNC group, $44.4 \% \pm 6.7 \%$; and control group, $34.4 \% \pm 5.4 \%(P<$ $.05)$. EF was only significant in the CCT group compared with that seen in the other 3 groups (Figure 1, a). LV end-systolic area was smaller in circumference after CCT compared with that seen in the control and BM-MNC groups and was also significantly smaller in the myoblast group compared with that seen in the control group.

Percentage fractional area shortening was significantly improved in the CCT group compared with that in all other groups (Figure 1,b). The myoblast group also showed improvement in cardiac performance, but this was not significant compared with that seen in the CCT group. The CCT group showed significant EF and fractional area shortening compared with that seen in the other groups. Enddiastolic area and end-systolic area was improved in the CCT and myoblast groups compared with that seen in the control group and showed no significant difference between the CCT and myoblast groups. There was no significant 

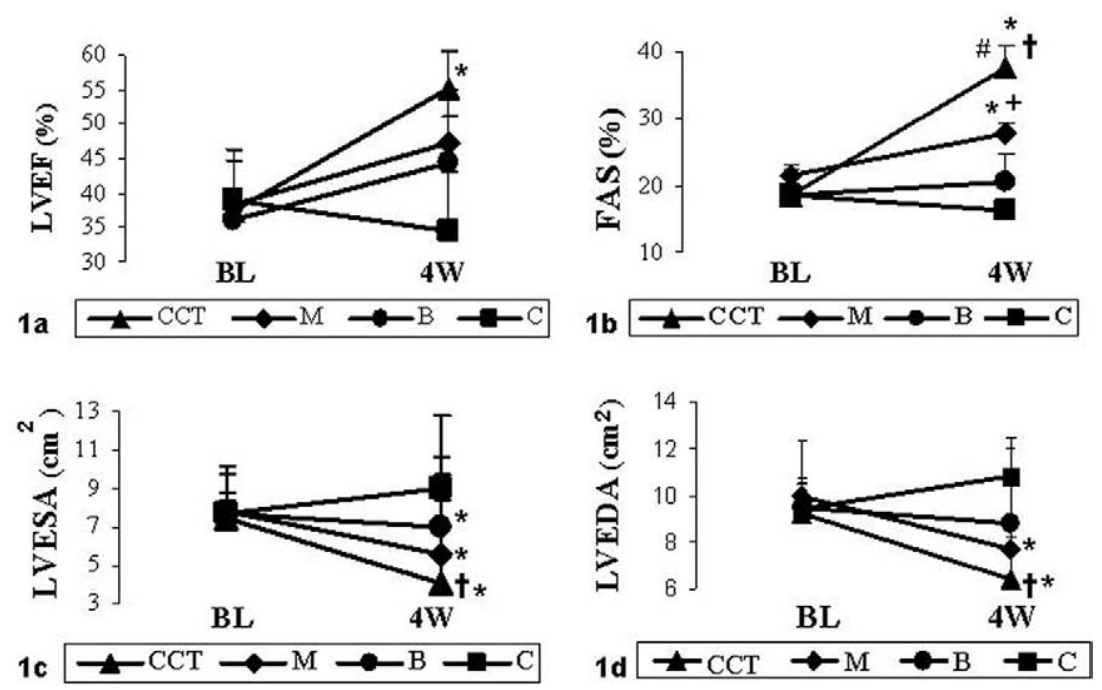

Figure 1. Echocardiography at baseline (2 weeks after the LAD ligation) and 4 weeks' follow-up after cell transplantation. CCT demonstrated improved left ventricular ejection fraction (LVEF; a), percentage fractional area shortening (FAS; b), left ventricular end-systolic area (LVESA; c), and left ventricular end-diastolic area (LVEDA; d). $M$, Myoblast group; $B$, BM-MNC group; $C$, control group. ${ }^{*} P<.05$ versus control; $\# P<.05$, CCT versus $M$; $+P<$ .05 , CCT versus $B ;+P<.05, M$ versus $B$. $B L$, Baseline; $4 W, 4$ weeks.

difference in improvement of cardiac function between the BM-MNC and control groups.

\section{Engrafted Myoblast Structure and Immunostaining}

Stained specimens (Figure 2) revealed grafts containing autologous skeletal muscle cells surviving within the scarred tissue, and this cellularity replaced the collagenous area. Hematoxylin and eosin staining clearly identified the grafted cells as myoblasts, which appeared in the form of multinucleated myofibers in the CCT and myoblast groups. These myofibers were all grafted parallel after host cell organization. Although a few surviving host cardiomyocytes in the scar area stained positive for MHC slow, both MHC fast and slow stained negative for SMs in the BM$\mathrm{MNC}$ and control groups.

After 4 weeks of cell transplantation, serial sections immunostained by directing against the fast-MHC isoform indicated the graft occupation by skeletal muscle structures, mainly fast-twitch fibers. A small percentage, approximately $20 \%$, stained positive for slow MHC, whereas approximately $40 \%$ of stained skeletal myofibers coexpressed the slow and fast MHC isoforms. The same sections stained negative with troponin $\mathrm{T}$, specific for cardiac, reconfirming their skeletal type identity (data not shown). No morphologic differences between the myoblasts of the CCT and myoblast groups were observed.

The measurements of graft diameter showed significantly large myoblast grafts in CCT group hearts compared with those of the myoblast group. The graft sizes emerged as follows: $0.31 \pm 0.09 \mathrm{~mm}^{2}$ in the CCT group versus 0.11 $\pm 0.05 \mathrm{~mm}^{2}$ in the myoblast group $(P<.05)$. On the basis of the histologic measurements, the ratio of infarct area to graft size area appeared to be around 1:1 in the CCT group versus 5:1 in the myoblast group.

\section{Effect of Cell Transplantation on Neovascularization} Immunostaining with antibodies to von Willebrand factor confirmed the endothelial character of these neovessels. Four groups were compared for neovascularization and counted as follows: CCT group, $45.6 \pm 12$; myoblast group, $26.5 \pm 8.1$; BM-MNC group, $30.7 \pm 15$; and control group, $7.1 \pm 1.4 \times 10^{2} / \mathrm{mm}^{2}(P<.05)$. Only the CCT group showed the highest number of new vessels and significant angiogenesis. Compared with the CCT group, the myoblast and BM-MNC groups showed almost half the number of vessels (Figure 3).

\section{Presence of Angiogenic Factors}

A markedly upregulated in vivo expression of VEGF was measured in hearts transplanted with CCT compared with those in the myoblast and BM-MNC groups $(2.9 \pm 0.7$ vs $0.24 \pm 0.7$ vs $1.9 \pm 0.2 \mathrm{ng} / \mathrm{g}$ tissue, respectively; $P<.05)$, but VEGF expression was not significantly higher in CCT in vitro cultures compared with that seen in myoblast and BM-MNC cultures.

In vivo HGF was also highly expressed after CCT compared with that seen in the myoblast and BM-MNC groups $(3.4 \pm 1.5$ vs $1.4 \pm 0.3$ vs $1.8 \pm 0.3 \mathrm{ng} / \mathrm{g}$ tissue, respec- 

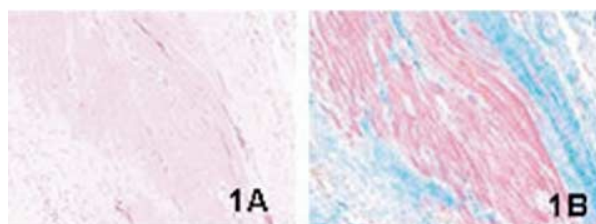

$2 A$

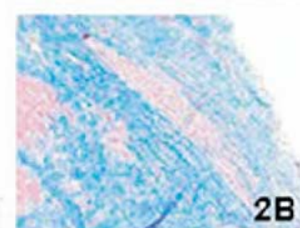

$3 A$

$4 A$

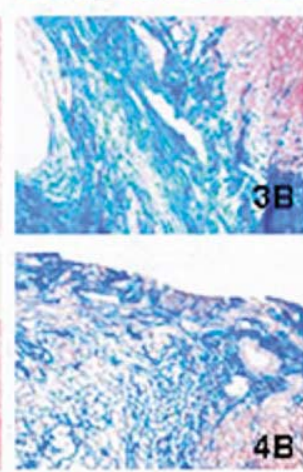

1C

1D

2C

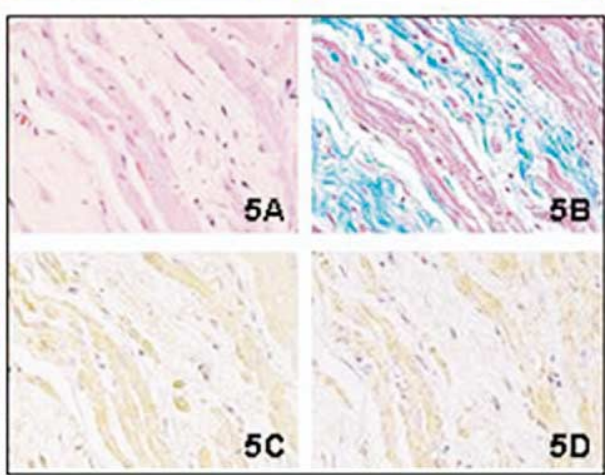

Figure 2. 1A-1D, CCT group; 2A-2D, myoblast group; 3A-3B, BM-MNC group; 4A-4B, control group at low magnification $(100 \times)$. 1A and $2 A$ shows survived grafted skeletal myofibers in the myocardial scar area in the CCT and myoblast groups. 1C and 2C show the fast-MHC expression by myoblasts, showing their skeletal muscle nature. 1D and 2D present slow-MHC expression, showing change in twitch from fast to slow. High magnification, $400 \times$, (5A-5D) demonstrates the elongated and multinuclear structure of myoblasts. 1A, 2A, 3A, 4A, and 5A, hematoxylin and eosin stain; 1B, 2B, 3B, 4B, and 5B, Masson trichrome stain; 1C, 2C, and 5C, MHC fast stain; 1D, 2D, and 5D, MHC slow stain.

tively; $P<.05$ ), and a similar expression pattern was observed in in vitro cultures. The in vivo bFGF expression level was lower but detectable in all 3 cell transplantation groups and was not significant between cell therapy groups. Infarcted hearts without any cell transplantation showed minimal detectable VEGF and bFGF expression (Figure 4).

\section{Discussion}

The current study demonstrates that the transplantation of autologous SMs induces further improved cardiac performance after the addition of BM-MNCs. The myoblasts attained their skeletal-type character after engrafting without showing phenotypic conversion to cardiomyocytes. The release of various growth factors was also highly expressed in the CCT group. We observed that the myoblasts combined with multipotent BM-MNCs are capable of surplus significant improvement in the deteriorated postinfarction LV function, with enhancement of cardiac performance and attenuation of cardiac remodeling.

After cell transplantation, the transplanted cells enter a new phase during which their destiny is still at stake. The in vivo environment plays a crucial role for the outcome of injected cells because they need an optimal microenvironment to home themselves. ${ }^{8}$ Cell transplantation with angiogenic factor gene transfection (ie, VEGF or HGF) showed better cardiac performance, ${ }^{10,17}$ but for clinical use, important issues like tumorigenesis, cell toxicity, immature vessel formation, and unregulated expression of transfected growth factors ${ }^{18}$ must be eliminated. Moreover, the release of cytokines from the transplanted cells is under strict physiologic control and occurs according to the in vivo microenvironment needs, and thus it is safer. ${ }^{19}$ Myoblasts and BMCs act as the natural supplier of HGF and VEGF, respectively, and provide feasible and safer therapy for clinical application.

A supporting CCT study of myoblasts and BM-MNCs in another small animal experiment by Ott and associates ${ }^{20}$ has shown the benefits of combining 2 potential cell lines for better cardiac performance and has also shown that the engraftment of SMs is more effective in combined transplantation of SMs and BM-MNCs compared with in isolated transplantation of SMs. One interesting finding of Ott 

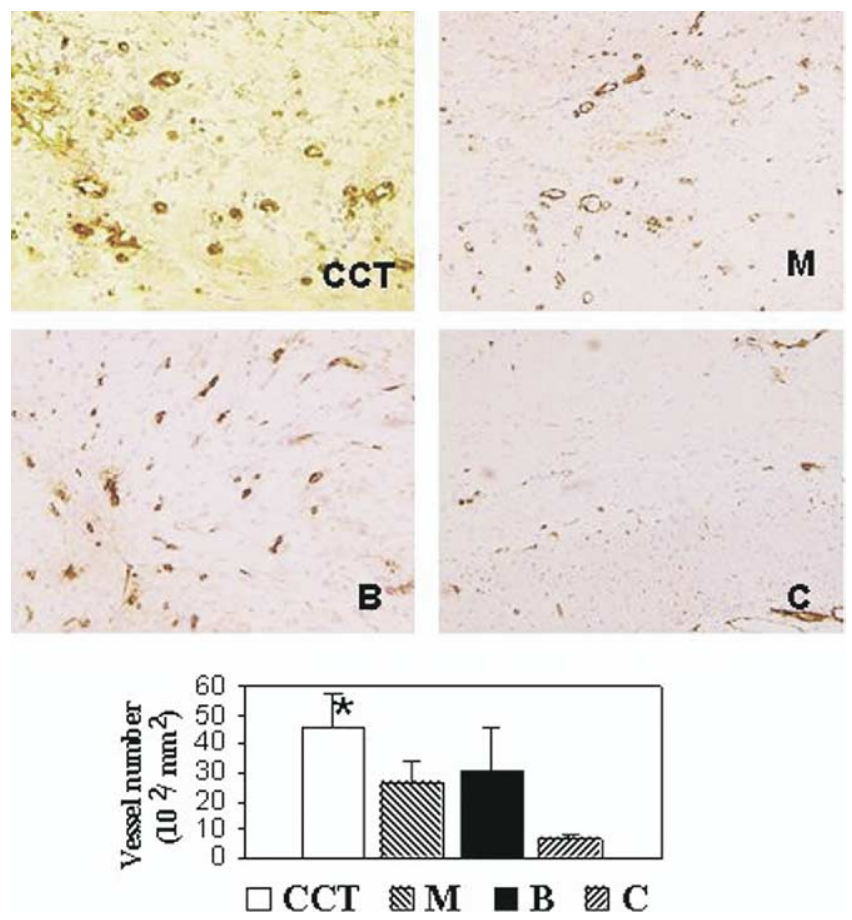

Figure 3. Staining with anti-factor VIII antibody for the endothelial cells. Capillary density comparison among the 4 groups showed a significantly higher number of vessels in the CCT group compared with numbers in the other cell transplantation groups (* $P<.05$ vs control). $M$, Myoblast group; $B$, BM-MNC group; $C$, control group.

and associates is that in combination with BM-MNCs $(5 \times$ $\left.10^{6}\right)$, half of the number of transplanted SMs $\left(5 \times 10^{6}\right)$ was as effective in restoring LV function and inducing angiogenesis as in isolated transplantation of SMs $\left(10^{7}\right)$. Similar to our study, their experiment did not show higher angiogenesis in the BMC transplantation group. Only our study has evaluated the expression of different growth factors and suggests it as one of the possible underlying mechanism for this effective combined therapy.

Our results suggest that a one-time dose of BMCs is capable of introducing significant angiogenesis, leading to better cardiac performance. The angiogenesis count was highest because of CCT and was widespread in and around the scar area. The reasoning for our results is that the endothelial cells and cytokines supplied by BM-MNCs improve the blood perfusion, ${ }^{11}$ thereby probably playing a pivotal role for better myoblast survival and heart function. The initial release of VEGF into the local environment in prolonging the survival of the cells that are not irreversibly damaged until angiogenesis is initiated might be the possible reason for early myoblast, as well as host cardiomyocyte, survival and is also probably helpful in retarding the further deterioration of ischemic heart. In addition, use of
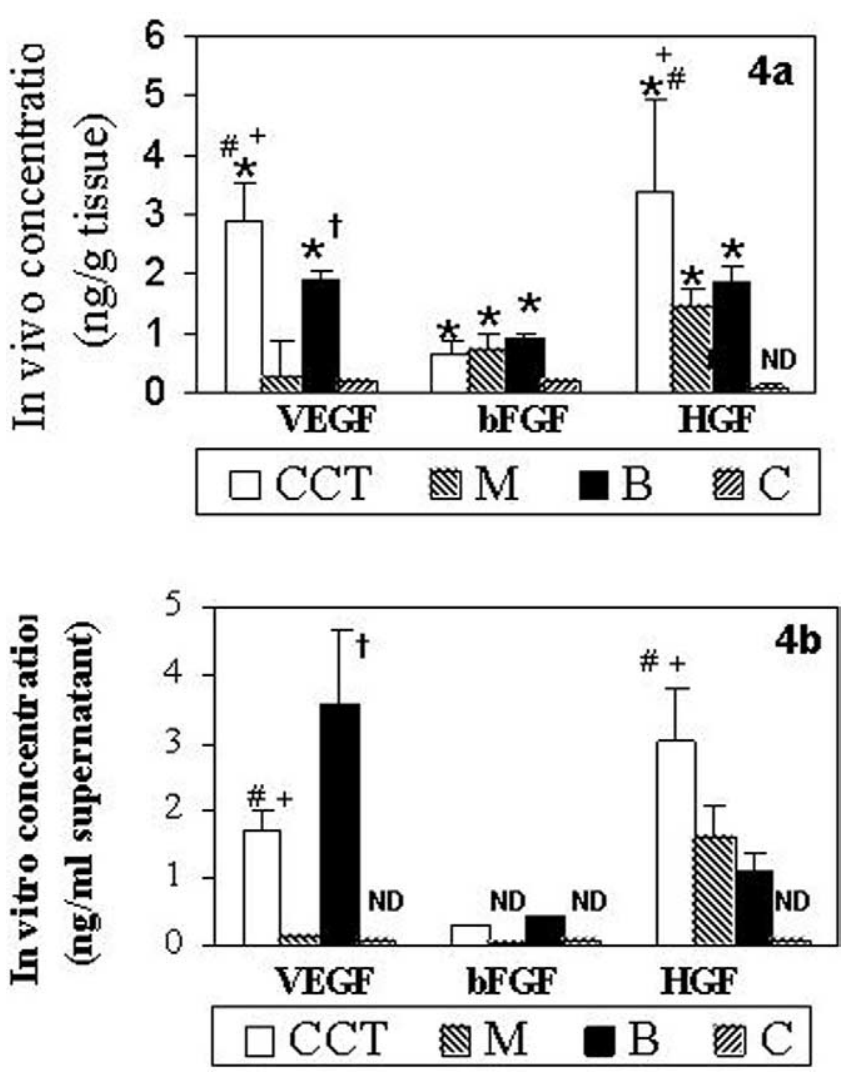

Figure 4. Detection of VEGF, bFGF, and HGF at day 5 after cell transplantation, as measured by means of ELISA in recipient rat hearts ( $\mathrm{n}=4$ in each group). $M$, Myoblast group; $B, B M-M N C$ group; $C$, control group; $N D$, less than the limit of detection. ${ }^{*} P<$ .05 versus control; $\# P<.05$, CCT versus $M ;+P<.05$, CCT versus $B ;+P<.05$, B versus $M$.

VEGF benefits myoblasts for their migration and survival ${ }^{21}$ and might be a vital support. Another available benefit is the release and use of $\mathrm{HGF}$, a unique angiogenic and fibrosispreventing factor, by the transplanted myoblasts for their own growth and recruitment of other cells. ${ }^{22}$ Although VEGF and HGF expression was higher in the CCT in vivo study, we are unaware of the exact physiologic reasons that might be involved in showing their opposite expression pattern in in vitro cultures. We believe that the response of cells (myoblasts and BM-MNCs) and the release of growth factors occur according to their microenvironment needs, and their cellular interaction might be an autoadjusting factor.

Grafted myoblasts accomplish the task of myogenesis, and this attained neomuscle mass plays a primary role in reremodeling the geometry of the LV chamber, as well as providing mechanical support. The grafted myoblasts in the CCT group mainly appeared in the form of large grafted bundles of myofibers and occupied the scar area wide- 
spread, whereas the myoblast group showed small-sized myoblast grafts. Recent reports mentioned the possible role of stromal cell-derived factor 1 in recruiting the stem cells. ${ }^{23}$ Because stromal cell-derived factor 1 is secreted in muscle tissue and muscle satellite cells express the functional CXCR4 receptors, ${ }^{24}$ they might chemoattract stem cells to the site of the need and might be beneficial for heart repair.

We found no contradiction with Reinecke and coworkers ${ }^{25}$ in observing the fate of transplanted SMs at 4 weeks. Our transplanted myoblasts expressed fast and slow skeletal MHC and did not transdifferentiate into cardiomyocytes after grafting. Because of deregulation and disappearance of intercalated disk protein (connexin 43) expression by grafted myoblasts in the mature stage, ${ }^{26}$ there is enough evidence for the absence of electromechanical coupling between host and grafted cells. ${ }^{27}$ Moreover, it has been reported that the transplanted BMCs do not induce electromechanical integration with host myocardium. ${ }^{28}$ Because SMs and BMCs do not form a gap junction in vivo, the chances that the cells will cause arrhythmia is also reduced.

One of the limitations of this study is justification of the comparison of adequate cell numbers in each group. The cell numbers among the CCT versus BM-MNC and myoblast versus BM-MNC groups are relatively different. However, such differences in numbers of cells between the CCT and myoblast groups is relatively small compared with the greater difference between their cardiac improvements, and this suggests that the efficiency and potentiality of CCT is far greater than just increasing the cell numbers of the same cell type.

In a previous clinical trial study, only $1.5 \times 10^{6} \mathrm{BM}$ cells were injected into patients undergoing coronary artery bypass grafting. The group showed improved perfusion, leading to better cardiac performance. ${ }^{29}$ Selection of $1 \times 10^{8}$ myoblasts and $3 \times 10^{6} \mathrm{BM}-\mathrm{MNCs}$ in our study is relevant to the number of cells used in the previous clinical trial studies. ${ }^{1,29}$

The limited number of BMCs and myoblasts used is to avoid the possibility of side effects, such as osteoformation by BMCs or arrhythmia by myoblasts. We confirmed negative osteoformation by calcium staining. High numbers of BMCs might release excessive growth factors and might lead to other calcification consequences. With $3 \times 10^{6}$ BM-MNCs transplanted, we observed no bone formation, suggesting an adequate cell supply. On the other hand, we did not detect arrhythmia in any of the myoblast-transplanted animals, as monitored by electrocardiography during echocardiography. Although the study by Menasché and colleagues $^{1}$ showed $8.7 \times 10^{8}$ myoblast transplantation to be arrythomogenic, another 2-year follow-up clinical study trial in 18 patients with a variable but lesser number of transplanted myoblasts $\left(10 \times 10^{6}\right.$ to $\left.3 \times 10^{8}\right)$ has shown myoblast transplantation to be a safe and nonarrhythmogenic therapy for ischemic cardiomyopathy. ${ }^{30}$ We believe that further increasing the number of myoblasts and $\mathrm{BMCs}$ is cautionary.

There are a few other limitations to our study. First, dogs are known to have native collateral vessels in the heart, and therefore it is possible that such native collateral formation might affect the functional recovery, but on the basis of the baseline (pretransplantation) cardiac performance values showing infarct formation in all groups, we believe that our overall conclusions were not distorted. In addition, although the coronary blood flow measurement is missing in our experiment, the absence of significant angiogenesis and lower growth factor expression in the BM-MNC and myoblast groups can be taken as supporting evidence for the ineffectiveness of native collateral vessels on results after cell transplantation. Second, we did not assess the size of infarction after cell transplantation. Third, the activation of the endogenous pathway for cardiac repair by growth factors from implanted cells is not measured and demonstrated. Further studies eliminating such drawbacks and examining BMC in vivo differentiation and their ability to generate phenotypes must be conducted.

Additional improvement with CCT is probably due to establishment and enrichment of the scar area with suitable microenvironment for the myoblasts and the host cardiomyocytes. This collaboration of different autologous cells and their growth factors should be appreciated and could be used for clinical benefit. Combined cellular cardiomyoplasty with SMs and BM-MNCs had been shown to be a novel myocardial repair strategy for treating ischemic heart failure.

We thank Shigeru Matsumi for his technical assistance.

\section{References}

1. Menasché P, Hagege AA, Vilquin JT, et al. Autologous skeletal myoblasts transplantation for severe postinfarction left ventricular dysfunction. J Am Coll Cardiol. 2003;41:1078-83.

2. Strauer BE, Brehm M, Zeus T, et al. Repair of infarcted myocardium by autologous intracoronary mononuclear bone marrow cell transplantation in humans. Circulation. 2002;106:1913-8.

3. Suzuki K, Murtuza B, Heslop L, et al. Single fibers of skeletal muscle as a novel graft for cell transplantation to the heart. J Thorac Cardiovasc Surg. 2002;123:984-93.

4. Taylor DA, Atkins BZ, Reedy MC, et al. Regenerating functional myocardium: Improved performance after skeletal myoblast transplantation. Nat Med. 1998;4:929-33.

5. Ghostine S, Carrion C, Souza LC, et al. Long-term efficacy of myoblast transplantation on regional structure and function after myocardial infarction. Circulation. 2002;106(suppl I):I131-6.

6. Murry CE, Whitney ML, Reinecke H. Muscle cell grafting for the treatment and prevention of heart failure. J Card Fail. 2002;8(suppl): S532-41.

7. Murry CE, Wiseman RW, Schwartz SM, Hauschka SD. Skeletal myoblast transplantation for repair of myocardial necrosis. J Clin Invest. 1996;98:2512-23. 
8. Suzuki K, Smolenski RT, Jayakumar J, et al. Heat shock treatment enhances graft cell survival in skeletal myoblast transplantation to the heart. Circulation. 2000;102(suppl):III216-21.

9. Retuerto MA, Schalch P, Patejunas G, et al. Angiogenic pretreatment improves the efficacy of cellular cardiomyoplasty performed with fetal cardiomyocyte implantation. J Thorac Cardiovasc Surg. 2004;127: 1041-9.

10. Miyagawa S, Sawa Y, Taketani S, et al. Myocardial regeneration therapy for heart failure. Hepatocyte growth factor enhances the effect of cellular cardiomyoplasty. Circulation. 2002;105:2556-61.

11. Kamihata H, Matsubara H, Mori $\mathrm{Y}$, et al. Implantation of bone marrow mononuclear cells into ischemic myocardium enhances collateral perfusion and regional function via side supply of angioblasts, angiogenic ligands, and cytokines. Circulation. 2001;104:1046-52.

12. Davani S, Marandin A, Mersin N, et al. Mesenchymal progenitor cells differentiate into an endothelial phenotype, enhance vascular density, and improve heart function in a rat cellular cardiomyoplasty model. Circulation. 2003;108(suppl II):II253-8.

13. Murry CE, Soonpaa MH, Bradford G, et al. Hematopoietic stem cells do not transdifferentiate into cardiac myocytes in myocardial infarcts. Nature. 2004;428:664-8.

14. Kocher AA, Schuster MD, Szabolcs MJ, et al. Neovascularization of ischemic myocardium by human bone-marrow-derived angioblasts prevents cardiomyocyte apoptosis reduces remodeling and improves cardiac function. Nat Med. 2001;7:430-6.

15. Perin EC, Dohmann HF, Vaughn WK, et al. Transendocardial, autologous bone marrow cell transplantation for severe, chronic ischemic heart failure. Circulation. 2003;107:2294-302.

16. Maruyama K, Mori Y, Murasawa S, et al. Interleukin-1 beta up regulates cardiac expression of vascular endothelial growth factor and its receptor $\mathrm{KDR} / \mathrm{flk}-1$ via activation of protein tyrosine kinases. $J \mathrm{Mol}$ Cell Cardiol. 1999;31:607-17.

17. Suzuki K, Murtuza B, Smolenski RT, et al. Cell transplantation for the treatment of acute myocardial infarction using vascular endothelial growth factor-expressing skeletal myoblasts. Circulation. 2001; 104(suppl I):I207-12.

18. Lee RJ, Springer ML, Blanco-Bose WE, et al. VEGF gene delivery to myocardium: deleterious effects of unregulated expression. Circulation. 2000;102:989-01.
19. Tang YL, Zhao Q, Zhang YC, et al. Autologous mesenchymal stem cell transplantation induce VEGF and neovascularization in ischemic myocardium. Regul Pept. 2004;117:3-10.

20. Ott HC, Bonaros N, Marksteiner R, et al. Combined transplantation of skeletal myoblasts and bone marrow stem cells for myocardial repair in rats. Eur J Cardiothorac Surg. 2004;25:627-34.

21. Germani A, Di Carlo A, Mangoni A, et al. Vascular endothelial growth factor modulates skeletal myoblast function. Am J Pathol. 2003;163: 1417-28.

22. Tatsumi R, Anderson JE, Nevoret CJ, et al. HGF/SF is present in normal adult skeletal muscle and is capable of activating satellite cells. Dev Biol. 1998;194:114-28.

23. Askari AT, Unzek S, Popovic ZB, et al. Effect of stromal-cell-derived factor 1 on the stem-cell homing and tissue regeneration in ischemic cardiomyopathy. Lancet. 2003;362:697-02.

24. Ratajczak MZ, Majka M, Kucia M, et al. Expression of functional CXCR4 by muscle satellite cells and secretion of SDF-1 by musclederived fibroblasts is associated with the presence of both muscle progenitors in bone marrow and hematopoietic stem/progenitor cells in muscles. Stem Cells. 2003;21:363-71.

25. Reinecke H, Poppa V, Murry CE. Skeletal muscle stem cells do not transdifferentiate into cardiomyocytes after cardiac grafting. $J \mathrm{Mol}$ Cell Cardiol. 2002;34:241-9.

26. Suzuki K, Brand NJ, Allen S, et al. Overexpression of connexin 43 in skeletal myoblasts: Relevance to cell transplantation to the heart. J Thorac Cardiovasc Surg. 2001;122:759-66.

27. Reinecke H, MacDonald GH, Hauschka SD, Murry CE. Electromechanical coupling between skeletal and cardiac, muscle: implications for the infarct repair. J Cell Biol. 2000;149:731-40.

28. Thompson RB, Emani SM, Davis BH, et al. Comparison of intracardiac cell transplantation: autologous skeletal myoblasts versus bone marrow cells. Circulation. 2003;108(suppl II):II264-71.

29. Stamm C, Westphal B, Kleine HD, et al. Autologous bone-marrow stem-cell transplantation for myocardial regeneration. Lancet. 2003; 361:45-6.

30. Nabil D, McCarthy P, Dinsmore J. Two-year follow-up of the safety and feasibility of autologous myoblasts transplantation in patients with ischemic cardiomyopathy: results from the United States experiences. Circulation. 2003;108:IV623. 